\title{
PT-Axisymmetric VCSELs with Linear Central Defect
}

\author{
Waqas W. Ahmed ${ }^{1}$, Muriel Botey ${ }^{1}$, Ramon Herrero ${ }^{1}$, and Kestutis Staliunas ${ }^{1,2}$ \\ 1 Departament de Física, Universitat Politècnica de Catalunya (UPC), Barcelona, \\ Spain \\ 2 Institució Catalana de Recercai Estudis Avançats (ICREA), Barcelona, Spain
}

\begin{abstract}
Semiconductor Lasers and particularly Vertical-Cavity Surface-Emitting Lasers (VCSELs) are important laser sources used for many purposes. However, the applications of these lasers are mainly restricted by their strongly multimode operation given by the lack of an intrinsic transverse mode selection mechanism [1]. The introduction of an axial PT-symmetric potential within this kind of lasers is expected to induce a field enhancement and localization at the symmetry axis, central part of the laser. The required complex potential, combining a modulated refractive index and gain-loss distributions, may be achieved by different configurations with actual fabrication techniques.

The Complex Ginzburg-Landau equation is used as a simple VCSELs model, and the numerical results show important localization effects; due to the asymmetric mode coupling energy converges to the center leading to a strong light confinement. The main consequence is a narrow and bright laser emission from the central part of the device. As the system nonlinearities introduce saturation limiting the maximum intensity of the output beam, the inclusion of a central linear defect in the structure allows a larger field concentration. Keywords: vertical-cavity surface-emitting lasers, PT-symmetry.
\end{abstract}

\section{INTRODUCTION}

Semiconductor Lasers are compact sources of coherent light used for many purposes. In particular VerticalCavity Surface-Emitting Lasers (VCSELs) are especially advantageous for their high light conversion efficiency, and low lasing threshold [1]. VCSELs usually present dynamical spatio-temporal chaotic behaviors arising from modulation instability and resulting in a poor spatial beam quality [2]. Moreover, the lack of an intrinsic transverse mode selection mechanism develops a deep multimode operation and for high enough pumping rates self-focusing, filamentation and spatial hole burning appear restricting the possible applications [3,4]. Usually considered stabilization methods are based on optical feedbacks that reduce robustness, compactness and applicability [5,6]. Moreover, these techniques enhance intensity noise and introduce negative temporal effects like linewidth broadening and coherence collapse [7]. However, Vertical External Cavity Surface Emitting Lasers (VECSELs) using an output spherical mirror are very common to ensure operation at the fundamental transverse mode. The monomode operation, although it strongly limits the size of the active region and the possible output power, allows a proper focalization and enables fibre coupling improving the applicability of these lasers [8]. Other methodologies directly act on the arising Modulation Instability to stabilize VCSELs [9].

Aside, the spatial modulation of the complex refractive index, i.e. refractive index and gain-loss modulations, has demonstrated strong capabilities to tailor light dispersion, including diffraction and diffusion, showing different spatial effects in linear light propagation along this kind of potentials [10-12]. Further, the same modulations in nonlinear systems introduce the management of spatial instabilities that intrinsically appear in laser systems and amplifiers $[9,13]$. One of the most interesting symmetries of complex spatial modulated materials is the PTsymmetry, where the real part of the modulation holds even symmetry while the imaginary part has odd symmetry. Therefore, the PT-symmetry condition requires that the complex refractive index, fulfils the condition: $n(r)=n *(-$ $r$ ). Parity-Time (PT-) symmetric materials are especially interesting in optics for supporting new properties and unusual light effects such modulations support a highly enhanced unidirectional light behavior around the so called PT-transition point, i.e. when index and gain-loss modulations are balanced [14-16]. Such modulations support a highly enhanced unidirectional light behavior around the so called PTtransition point, i.e. when index and gainloss modulations are balanced [14-16]. Unidirectional light coupling may be achieved by periodic PT-symmetric materials in which the index and gain-loss modulations are dephased a quarter of the spatial period of the modulation introducing an asymmetric coupling between light transverse modes. By an analogous periodic axial PT-symmetric potential, light fields may be expected to be localized at a desired point, the symmetry axis [17]. In this case the inwards radial coupling would be responsible of directing to the central point of the structure obtaining important localization effects (see Fig. 1).

In linear systems, light propagation through PT-axisymmetric potentials creates an extreme localization at $r=$ 0 , accompanied by a strong field enhancement, exhibiting an exponential temporal growth. The localization of light in a central beam with small width and high intensity opens the possibility to obtain laser light sources with very narrow and bright beams from lasers. However, the application of such potentials to laser models, and in particular to the Complex Ginzburg-Landau equation as a simplified VCSEL model, usually shows typical turbulent states with no field concentration. The field grows all over the system and nonlinearities introduce saturation limiting the maximum intensity of the output beam. As a consequence the field cannot be localized and 
field becomes turbulent with typical saturation amplitude. The inclusion of a central linear defect in the structure allows the field concentration.

We explore the PT-axisymmetric laser with a central linear defect in 1D and 2D transverse dimensions, scanning the main parameters as laser pump, linewidth enhancement factor of the semiconductor, amplitude of the PT-modulated potential, i.e. refractive index amplitude and gain-loss amplitude, as well as the width of the defect. Results show field concentrations significantly larger than the saturated laser intensity with stationary behaviours and pulsed regimes. Finally, we include losses in the central part of the VCSEL model to consider the field coupling to an output fibre.

\section{VCSEL MODEL WITH PT-AXISYMMETRIC COMPLEX INDEX AND CENTAL DEFECT}

In the simplest approximation, the field dynamics of VCSELs can be described by complex Ginzburg-Landau equation:

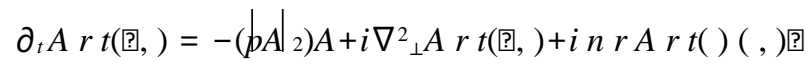

where $A(r, t)$ is the envelope of complex field distributed in space, $p$ is the pump parameter and the nonlinearity is due to gain saturation. For simplicity, we use normalized spatial and temporal coordinates.

The complex refractive index modulation of Fig.1a presents a simple 1D harmonic modulation with a PTaxisymmetric profile. The symmetry axis is located at $x=0$ and real and imaginary parts are dephased by $\pm \pi / 2$ yielding to a unidirectional coupling with opposite directions for $x<0$ and $x>0$. In this 1D case the complex refractive index can be expressed as:

$$
\left.\left.n x()=n \operatorname{Cos} x_{\operatorname{Re}} \mid+-\phi\right) \text { in } \operatorname{Sin} x_{\operatorname{Im}} \mid+\phi\right)
$$

where $n_{\text {Re }}$ denotes the normalized amplitude of real refractive index, and $n_{\text {Im }}$ the normalized amplitude of the gainloss modulation. The ratio between both amplitudes is expected to confer the asymmetric character to the system. Besides, the phase $\varphi$, characterizes the potential at the symmetry centre, which we assume as $\varphi=0$ [17]

(a)

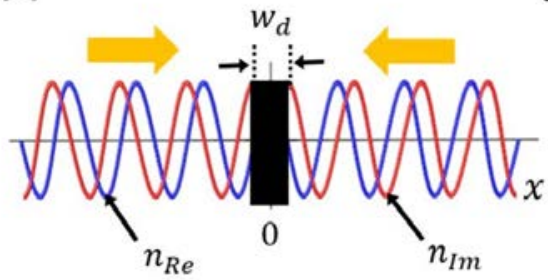

(b)

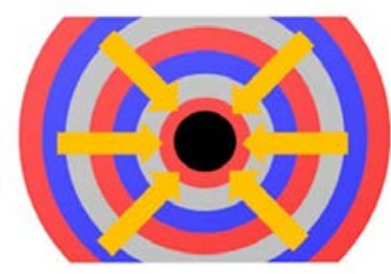

(c)
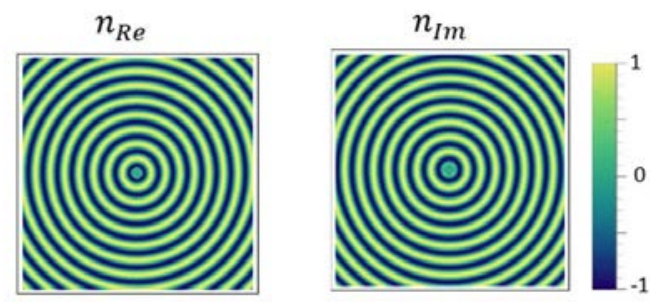

Figure 1: (a) 1D PT-axisymmetric complex optical potential, where $n_{R e}$ is the real index modulation, and $n_{\text {Im }}$ is the gain-loss modulation with a linear central defect of width $w_{d}$. Field concentration is obtained inside the linear defect; (b) 2D analogous PT-axisymmetric configuration, where the arrows indicate the inwards coupling; (c) Refractive index $n_{R e}$ and gain-loss $n_{I m}$ modulations in the $2 D$ case.

In 2D, the number of possible PT-symmetric geometries are enlarged [18]. Here, we just consider the PTsymmetric radial configuration:

$$
n r()=n \operatorname{Cos} r_{\mathrm{Re}}(+-\phi) \text { in } \operatorname{Sin} r_{\mathrm{Im}}
$$

where the coupling between inward and outward waves becomes asymmetric (see Fig. 1b). 


\section{LIGHT LOCALIZATION AND ENHANCEMENT}

First, we investigate the 1D system, by numerically solving Eq. (1) with the complex refractive index defined by Eq. (2a). We numerically integrate the paraxial wave equation using a split step method, for a Gaussian initial beam in the form: $A x()=e^{\left(-x w_{2} / 2\right)}$ where $w$ is the width of the initial beam profile. Typically, the field in the laser system is uniformly amplified by the gain and the maximum intensity of the output beam is restricted to the normalized pump intensity, $p$, due to saturation phenomena. In our study, the inclusion of a central linear defect in the structure avoids saturation around axis and allows a significantly larger field concentration and enhancement than in conventional laser systems. Such PT-axisymmetric systems develop extraordinary field localization on axis and the peak intensity becomes significantly larger than the normalized pump after a sufficient evolution time. We explore the parameter space $\left(n_{\mathrm{Re}}, n_{\mathrm{Im}}\right)$ for a fixed defect width and identify the possible operating regimes. Stationary, oscillatory or pulsed regimes can take place, showing different spatiotemporal dynamics. Figure 2 describes the possible laser dynamics for a defect width $w_{d}=2$ depending on the modulation amplitudes of the complex refractive index $\left(n_{\mathrm{Re}}, n_{\mathrm{Im}}\right)$. The average intensity of the central peak and the amplitude of the temporal oscillations are calculated in this parameter space to characterize the stationary and oscillatory regimes.

(a)

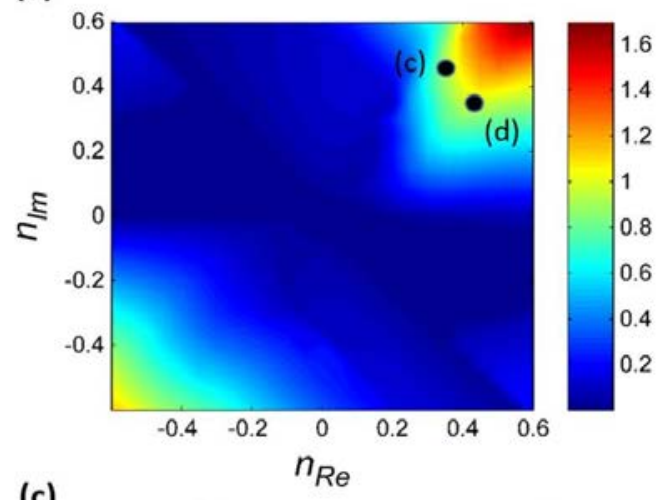

(c)

(i)

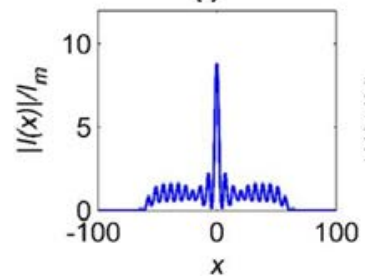

(ii)

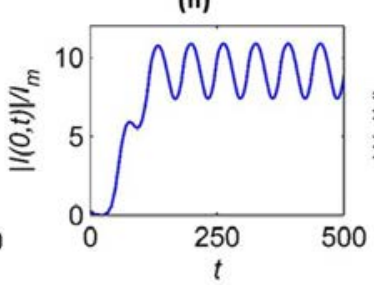

(b)

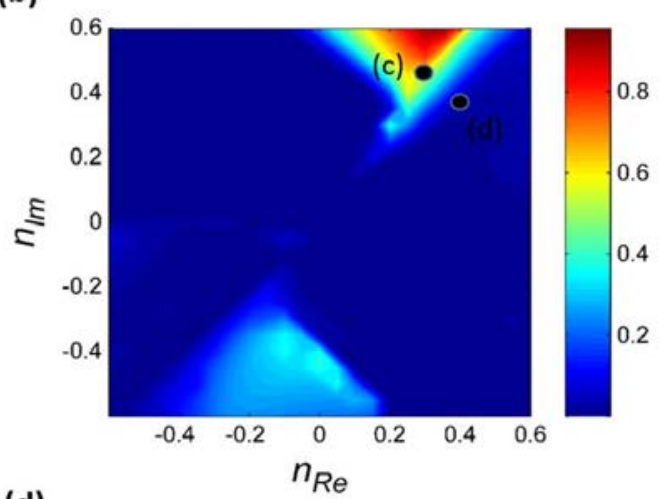

(d)

(i)

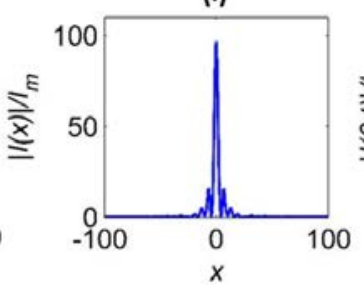

(ii)

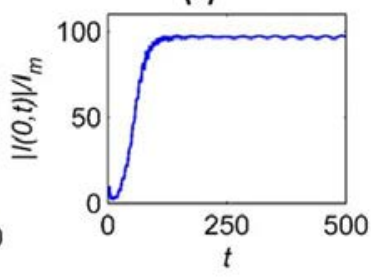

Figure 3: (a) Average normalized amplitude of the central peak intensity and (b) Amplitude of the temporal oscillation for a pump $p=0.01$ and a defect width $w_{d}=2$. Stationary regimes correspond to blue while dark red values correspond to large amplitude oscillations. The two representative points marked as (c) with parameter set $\left(n_{R e}=0.35, n_{I m}=0.45\right)$, and (d) with parameters $(0.4,0.35)$, correspond to an oscillatory and to a stationary state, respectively. In both cases, the spatial intensity profiles and evolution of central average peak intensity normalized to the mean intensity, $I_{m}$, are shown in (i) and (ii), respectively, revealing that intensity is increased by two orders of magnitude.

The calculated maps for average intensity and amplitude of temporal oscillation built in parameter space are shown in Fig. 2a and Fig. 2b, respectively. We observe that the field is accumatled at the top right and bottom left regions, where the coupling is inward for both left and right half space. The relative amplitudes of index and gainloss modulations are clue parameters to obtain maximum intensity enhancement. The stationary and oscillatory regimes mainly depend on the relative modulation amplitudes. At the PT-phase transition $\left(n_{\mathrm{Re}}=n_{\mathrm{Im}}\right)$, the stationary regime becomes unstable through a supercritic Hopf bifurcation arising the oscillatory regime. To illustrate the effect of the PT-potential on the spatio-temporal field dynamics, we consider two representative points, 2c and 2d, with stationary and oscillatory regimes, respectively. The intensity profiles and evolution of peak intensity normalized with the mean intensity, $\mathrm{I}_{\mathrm{m}}$, for the corresponding points are provided in Fig. 2c and Fig. 2dThe periodic oscillations for (c) clearly indicate the pulsating temporal behaviour for $n_{\mathrm{Re}}<n_{\mathrm{Im}}$ in top right and bottom left corners.

We finally analyze the 2D PT-axisymmetric laser system with $n(r)$ defined by Eq. (2b). The results for particular parameter sets are provided in Fig. 3. We observe a strong field enhancement at $r=0$ clearly attributed to the asymmetric radial coupling between inward and outward propagation waves. The axial cross-sectional profile of the intensity and evolution of central peak intensity are show in Fig. 3a and Fig. 3b, respectively. In the 2D geometry case, the results are analogous to the simple 1D case, although stronger localization effects are expected. In this work we have analyzed the radial geometry although the 2D transvers space allow different geometries to 
be studied. Moreover, the same PT-axisymmetric geometry of the complex potential is suitable to be implemented in different broad area laser systems.

(a)

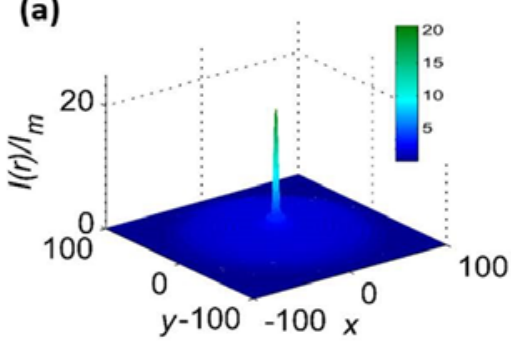

(b)

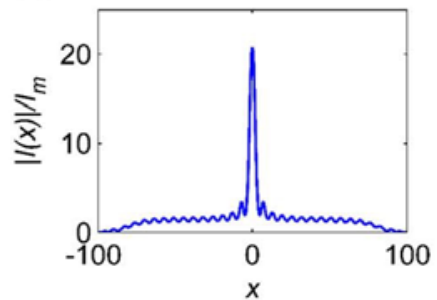

(c)

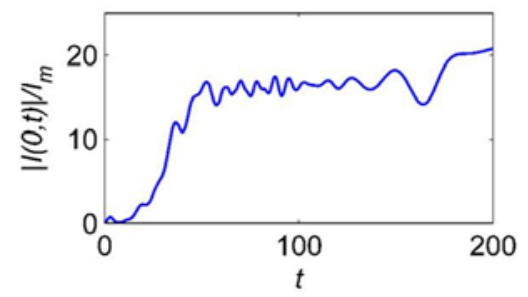

Figure 3: (a) Normalized intensity profile after long time evolution for a normalized pump $p=0.05$, and amplitudes normalized complex index $n_{R e}=n_{I m}=0.3$; (b) Axial cross-section of the intensity; (c) Intensity evolution of the onaxis intensity for a long time evolution.

\section{CONCLUSIONS}

We propose a novel class of PT-axisymmetric VCSELs which may serve as a narrow and bright output light source. The idea is based on axial PT-symmetry with central defect area in structure to avoid saturation and allows a larger field concentration. We studied the parameter dependence of the intensity enhancement depending on relative amplitudes of refractive index and gain-loss modulation and width of the central defect. We essentially identify two distinct operating regimes i.e., stationary and oscillatory regimes for such lasers depending on the modulation amplitudes. We observe a significant enhancement in the output intensity in our case as compared to pump laser intensity. Our proposal is generic in nature and could be applied to other broad aperture lasers or amplifiers for the regularization of the spatial structure the output radiation.

\section{REFERENCES}

[1] K. Iga, F. Koyama, and S. Kinoshita, IEEE J. Quantum Electron. 24, 1845 (1988).

[2] J. Ohtsubo, Semiconductor Lasers: Stability, Instability and Chaos, 3rd ed. (Springer, Berlin, 2013).

[3] K. Hirose, Y. Liang, Y. Kurosaka, A.Watanabe, T.Sugiyama, and S. Noda, Nat. Photonics 8, 406 (2014).

[4] J. R. Marciante and G. P. Agrawal, IEEE J. Quantum Electron. 32, 590 (1996)

[5] S. Takimoto, T. Tachikawa, R. Shogenji, and J. Ohtsubo, IEEE Photon. Technol. Lett. 21, 1051 (2009). [6] S. K. Mandre, I. Fischer, and W. Elsäßer, Opt. Lett. 28, 1135 (2003).

[7] J. Y. Law and G. P. Agrawal, J. Opt. Soc. Am. B. 15, 562 (1998).

[8] A.C. Tropper and S. Hoogland, J. Quantum Electron. 30, 43 (2006).

[9] W. W. Ahmed, S. Kumar, R. Herrero, M. Botey, M. Radziunas, and K. Staliunas, Phys. Rev. A 92, 043829 (2015).

[10] K. Staliunas, R, Herrero, and R. Vilaseca, Phys. Rev. A 80, 013821 (2009).

[11] M. Botey, R. Herrero, and K. Staliunas, Phys. Rev. A 82, 013828 (2010).

[12] R. Herrero, M. Botey, and K. Staliunas, Phys. Rev. A 89, 063811 (2014).

[13] S. Kumar, R. Herrero, M. Botey, and K. Staliunas, Optics Letters, 39, 5598-5601 (2014).

[14] A. Guo, G. J. Salamo, D. Duchesne , R. Morandotti, V. Aimez, G.A. Siviloglou, and D.N. Christodoulides, Phys. Rev. Lett. 103, 093902 (2009).

[15] L. Feng, Y.-L. Xu, W. S. Fegadolli, M.-H. Lu, J. E. Oliveira, V.R. Almeida, Y.-F. Chen, and A. Scherer, Nat. Mater. 12, 108 ( 2012).

[16] M. Turduev, M. Botey, I. Giden, R. Herrero, H. Kurt, E. Ozbay, and K. Staliunas, Phy. Rev. A 91, 023825 (2015).

[17] W. W. Ahmed, R. Herrero, M. Botey, and K. Staliunas, Phys. Rev. A 94, 053819 (2016).

[18] W. W. Ahmed, R. Herrero, M. Botey, and K. Staliunas, Phys. Rev. A accepted for publication (2017). 